\title{
PROPAGATION OF THE MEASUREMENT ERRORS AND MEASURED MEANS OF PHYSICAL QUANTITIES FOR THE ELEMENTARY FUNCTIONS $x^{2}$ AND $\sqrt{x}$
}

\begin{abstract}
Rules for the propagation of the error and mean value obtained for a measured physical quantity $x$ onto another one, which is coupled to the former by means of the $x^{2}$ or $\sqrt{x}$ functional relation, have been derived. Those rules are inherently based on the Gaussian weight scheme, so that they should provide correct results in the framework of the latter with discrete data, which is typical of a real physical experiment (with samplings). The obtained analytical form that represents the mentioned rules (the "analytical propagation rules") and their exact character allow the processing and analysis of experimental data to be simplified and accelerated.

Ke ywords: propagation of error, propagation of uncertainty.
\end{abstract}

\section{Introduction}

This work is devoted to a challenging problem dealing with the estimation of the errors for physical quantities obtained at indirect measurements. In such a way, it proposes a solution for some part of a more general problem concerning the error propagation. In more details, the problem of error propagation was described in works $[1,2]$.

There are two approaches to solve this problem. All modern theoretical and practical applications, as well as calculation procedures and developments, consider the error propagation exclusively on the basis of the Taylor series expansion (differentiation) [412]. The scope of problems aimed at the "analytical" error propagation was described, best of all, in work [1]. Some efforts in this direction are made in this work, which is a logic continuation of work [2]. Namely, two widespread elementary functions, $x^{2}$ and $\sqrt{x}$, are considered.

(C) G.G. RODE, 2017

184

\section{New Rules of Calculation} of the Mean Values and the Error Propagation for the Elementary Functions $x^{2}$ and $\sqrt{x}$

In order to derive analytical rules for two chosen functions $\left(x^{2}\right.$ and $\left.\sqrt{x}\right)$, the mean $x_{\text {mean }}$ and the "error" $k(\Delta x)_{\text {mean }}^{2}$ were related (formalized) to the basic concepts of mathematical statistics [2]:

$x_{\text {mean }} \approx E_{x}, \quad k(\Delta x)_{\text {mean }}^{2} \approx D_{x}$,

where $E_{x}$ is the mathematical expectation, and $D_{x}$ the dispersion of a measured quantity $x$. According to work [1], those parameters are defined as follows:

$$
\begin{aligned}
& \mu=E_{x}=\int_{-\infty}^{\infty} x f(x) d x \\
& D_{x}=\int_{-\infty}^{\infty}\left(x-E_{x}\right)^{2} f(x) d x=\int_{-\infty}^{\infty}(x-\mu)^{2} f(x) d x \\
& \int_{-\infty}^{\infty} f(x) d x=1 .
\end{aligned}
$$


For the functional relation $y=h(x)$, the mathematical expectation $E_{h}$ and the dispersion $D_{h}$ are defined as follows $[1,2]$ :

$$
\begin{aligned}
& \chi=E_{h}=\int_{-\infty}^{\infty} h(x) f(x) d x \\
& D_{h}=\int_{-\infty}^{\infty} h^{2}(x) f(x) d x-E_{h}^{2} .
\end{aligned}
$$

Among the distributions $f(x)$, the so-called normal (Gaussian) probability distribution is considered to be the most important $[1,2]$ :

$f(x)=\frac{p}{\sqrt{\pi}} \exp \left[-p^{2}(x-\mu)^{2}\right]$

where

$p^{2}=\frac{1}{2 D_{x}}, \quad \mu=E_{x}$.

In Eqs. (2) and (3), the quantities $\mu=E_{x}$ and $D_{x}$ enter the function $f(x)$ as its parameters. Therefore, strictly speaking, $f(x)$ can be written as $f\left(x, E_{x}, D_{x}\right)$ :

$E_{h}=\int_{-\infty}^{\infty} h(x) f\left(x, E_{x}, D_{x}\right) d x$,
$D_{h}+E_{h}^{2}=\int_{-\infty}^{\infty} h^{2}(x) f\left(x, E_{x}, D_{x}\right) d x$.

It is easy to notice that Eqs. (4) and (5) are integral equations. By solving them, we could obtain a required analytical relation between $E_{h}$ and $D_{h}$ (they are mean analogs for the function $h(x)$ ), on the one hand, and $E_{x}$ and $D_{x}$ (the analogs of the measured means), on the other hand. It turned out, that it is possible to select tabulated integrals [3] that are similar to expressions (4) and (5) and, in this manner, to solve the problem for two elementary functions, $x^{2}$ and $\sqrt{x}$ (see Appendix).

As a result, for the function $x^{2}$, the mentioned relations look like

$$
\begin{aligned}
& E_{h}=E_{x^{2}}=E_{x}^{2}+D_{x} ; \\
& D_{h}=D_{x^{2}}=2 D_{x}^{2}+4 E_{x}^{2} D_{x},
\end{aligned}
$$

where $E_{x}$ and $D_{x}$ correspond to the mean and variance, respectively, of measured data, and $E_{x^{2}}$ and
$D_{x^{2}}$ to the mean and variance, respectively, of the measured result propagation, by using the function $x^{2}$. For the function $\sqrt{x}$, the relations look like

$$
\begin{aligned}
& E_{h}^{4}=E_{\sqrt{x}}^{4}=E_{x}^{2}-\frac{1}{2} D_{x} \\
& D_{h}=D_{\sqrt{x}}=E_{x}-\sqrt{E_{x}^{2}-\frac{1}{2} D_{x}},
\end{aligned}
$$

where $E_{x}$ and $D_{x}$ correspond to the mean and the variance of measured data, respectively, and $E_{\sqrt{x}}$ and $D_{\sqrt{x}}$ to the mean and the variance of the measured result propagation, by using the function $\sqrt{x}$.

Hence, we obtained (see Appendix) the required rules for the "error propagation" and the calculation of the "shifted mean" of the type $E_{h}=E_{h}\left(E_{x}, D_{x}\right)$ and $D_{h}=D_{h}\left(E_{x}, D_{x}\right)$ for the functions $h(x)=x^{2}$ and $h(x)=\sqrt{x}$.

\section{Application of New Rules to Experimental Data}

Let a set of experimental data include separate random values $x_{i}$ obtained for the measured physical quantity $x$. This is the so-called sample $\left\{x_{i}\right\}$. The quantity $x$ can be characterized by a continuous distribution [1]. In other words, these are values that were randomly "chosen by a device" from a continuous set of values.

Let us consider how the obtained relations are obeyed in the case of samples. For this purpose, let us calculate the means for four samples using the standard procedure (it will be regarded as a reference one): for two experimental data sets $\left\{x_{i}\right\}$ and two sets of calculated functions $x^{2}$ and $\sqrt{x}$. Then we intend to compare them with the results obtained, by using relations (6) and (7).

\section{Example for $x^{2}$}

As an example for $x$, let us take the sample $\left\{x_{i}\right\}$ consisting of 20 measurements for one of the unit cell parameters in the crystal lattice:

$\left\{x_{i}\right\}=9.75,9.778,9.792,9.841,9.841,9.848,9.848$, 9.855, 9.855, 9.862, 9.722, 9.708, 9.659, 9.659, 9.652, $9.652,9.645,9.645,9.638,9.75(\AA)$;

Hereafter, the samples are constructed on the basis of measurement data obtained on a three-circle diffractometer $[13,14]$. The arithmetic means calculated for 


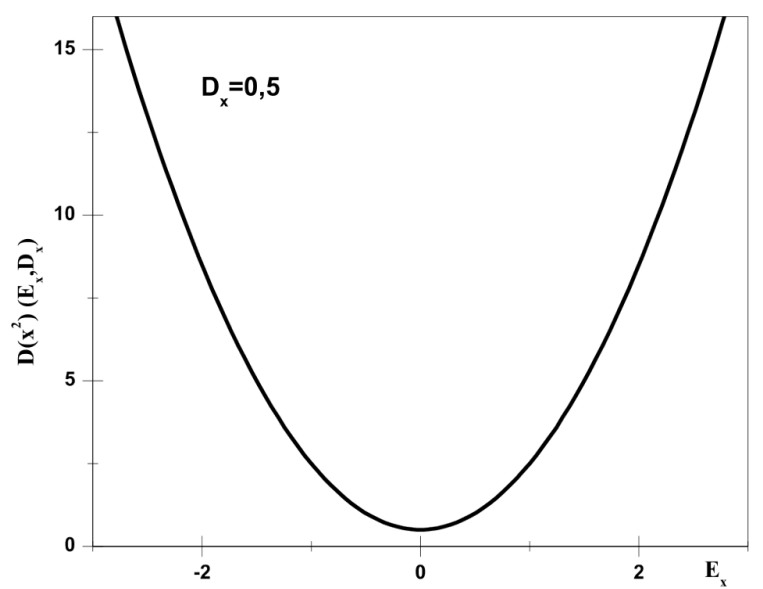

Fig. 1. Dependence of the $D_{x^{2}}\left(E_{x}, D_{x}\right)$ function variance on $E_{x}$ at $D_{x}=0.5$

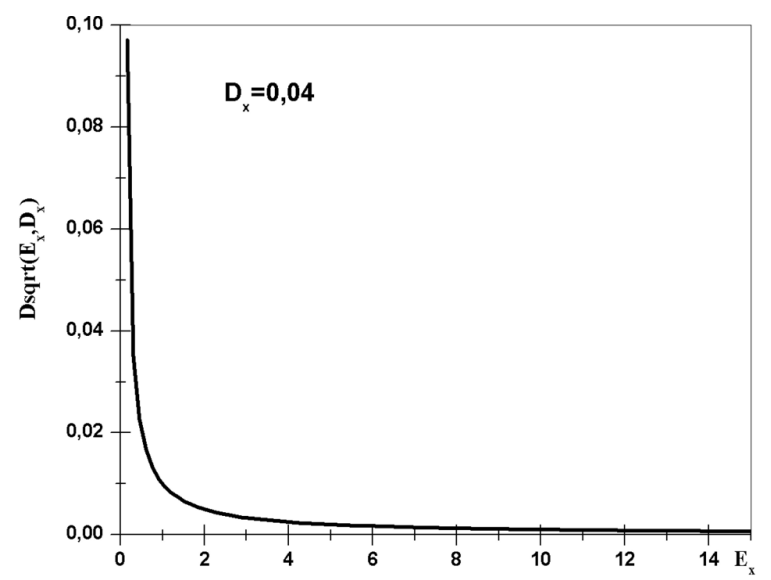

Fig. 2. Dependence of the $D_{\sqrt{x}}\left(E_{x}, D_{x}\right)$ function variance on $E_{x}$ at $D_{x}=0.04$

this sample with the constant probability $w_{i}=1 / 20$ give the following values:

$E_{n}=9.75 ; \quad D_{n}=0.00729, \quad \Delta_{n}=0.08945$.

Using them as the first approximation, we can calculate the Gaussian means (this routine takes 2 to 3 iterations) with the help of the Gaussian weight scheme

$$
\begin{aligned}
E_{x} & =\sum x_{i} f_{i} ; \quad D_{x}=\left(\sum x_{i}-E_{x}\right)^{2} f_{i} ; \\
\Delta_{x} & =\sqrt{D_{x}}
\end{aligned}
$$

where $f_{i}$ is the probability over the sample normalized to 1 ,

$$
f_{i}=\frac{w_{i}}{\sum_{n} w_{i}}, \quad \sum_{n} f_{i}=1,
$$

\section{6}

and

$w_{i}=\frac{p}{\sqrt{\pi}} \exp \left[-p^{2}\left(x_{i}-\mu\right)^{2}\right], \quad p^{2}=\frac{1}{2 D_{x}}$.

As a result, we obtain

$E_{x}=9.75, \quad D_{x}=0.00537, \quad \Delta_{x}=0.07328$.

In other words, for this sample, we have $E_{x}=$ $=9.75 \pm 0.07$.

In order to correctly calculate the means for the function $x^{2}$, it is necessary to construct a new statistical sample $\left\{x_{i}^{2}\right\}$ and to calculate the means for it. The new sample is

$\left\{x_{i}^{2}\right\}=95.0625,95.60928,95.88326,96.84528$,

96.84528, 96.9831, 96.9831, 97.12103, 97.12103,

97.25904, 95.0625, 94.51728, 94.24526, 93.29628,

93.29628, 93.1611, 93.1611, 93.02602, 93.02602,

$92.89104\left(\AA^{2}\right)$.

The corresponding arithmetic means are

$E_{n}=95.06979, \quad D_{n}=2.7725, \quad \Delta_{n}=1.6651$.

Using the values obtained for $E_{x}, D_{x}$, and $\Delta_{x}$, and carrying out the calculations in accordance with formulas (8) and (9), we obtain the sought means for the function $x^{2}$ in the standard way:

$E_{x^{2}}=95.06786, \quad D_{x^{2}}=2.04139, \quad \Delta_{x^{2}}=1.42877$.

The "propagation of errors" with the help of relations (6) brings about the following values:

$E_{x^{2}}=95.06787, \quad D_{x^{2}}=2.042, \quad \Delta_{x^{2}}=1.42899$.

Hereafter, we intentionally left more digits than required (in particular, two digits for $D_{x}$, and only one for $\Delta_{x}$ ) in order to trace all calculations in more details. Comparing the results, we may assert that, in the case of the function $x^{2}$, the standard deviations $\Delta_{x^{2}}$ completely coincide. In other words, the propagation of errors for the function $x^{2}$ with the use of relations (6) is proper and provides good results for samples.

\section{Example for $\sqrt{\boldsymbol{x}}$}

Now, let us consider an example for the function $\sqrt{x}$. The following sample of measurement data ob-

ISSN 2071-0194. Ukr. J. Phys. 2017. Vol. 62, No. 2 
tained for the parameter $a^{2}$ of a unit cell will be used:

$\left\{y_{i}\right\}=\left\{x_{i}^{2}\right\}=40.45,40.895,40.984,41.518,41.518$, 41.607, 41.607, 41.696, 41.785, 41.785, 40.45, 40.005, $39.916,39.382,39.382,39.293,39.293,39.204$,

$39.115,39.115\left(\AA^{2}\right)$.

According to the standard calculation scheme used above for the sample means,

i) first, we calculate the arithmetic means (the probability $\left.w_{i}=1 / 20\right)$,

$E_{n}=40.45, \quad D_{n}=1.05587, \quad \Delta_{n}=1.02756 ;$

ii) then, we calculate the Gaussian means with the weight scheme (9),

$E_{y}=40.45, \quad D_{y}=0.79847, \quad \Delta_{y}=0.89357$

iii) further, we create an array (sample) for the function $\sqrt{y}$,

$\left\{\sqrt{y_{i}}\right\}=6.36003,6.39492,, 6.40187,6.44345$,

6.44345, 6.45035, 6.45035, 6.45724, 6.46413, 6.46413,

6.36003, 6.32495, 6.31791, 6.27551, 6.27551,

$6.26841,6.26841,6.26131,6.2542,6.2542(\AA)$.

The corresponding arithmetic means are

$E_{\sqrt{y}}=6.35952, \quad D_{\sqrt{y}}=0.00653, \quad \Delta_{\sqrt{y}}=0.08081$.

The statistical treatment of this sample with the use of the values of $E_{y}, D_{y}$, and $\Delta_{y}$ gives us

$E_{\sqrt{y}}=6.35965, \quad D_{\sqrt{y}}=0.00494, \quad \Delta_{\sqrt{y}}=0.07029$.

The calculations according to relations (11) result in the following values of "error propagation":

$E_{\sqrt{y}}=6.35964, \quad D_{\sqrt{y}}=0.00494, \quad \Delta_{\sqrt{y}}=0.07025$.

One can see that the coincidence is ideal in this case. In other words, in the case of the function $\sqrt{x}$, the "propagation of errors" by formulas (7) is proper and works well for samples.

It should be noted that the correctness of the inverse formula should be verified, by using samples with wider intervals of means and dispersions. However, this task goes beyond the scope of this paper.

ISSN 2071-0194. Ukr. J. Phys. 2017. Vol. 62, No. 2

\section{Some Common Properties of the Obtained Relations}

The analytical form obtained for the propagation rules makes it possible to easily distinguish the peculiarities of corresponding relations and even plot graphic dependences, which is very useful, when planning and analyzing a physical experiment.

It should be emphasized that the quantities $E_{h}, D_{h}, E_{x}$, and $D_{x}$ are interrelated with one another. In addition, $E_{h}$ and $D_{h}$ are functions of two variables rather than one:

$E_{h}=E_{h}\left(E_{x}, D_{x}\right), \quad D_{h}=D_{h}\left(E_{x}, D_{x}\right)$.

Sometimes, this fact may be difficult to get used to, e.g., like the fact that the variances $\Delta\left(x^{2}\right)$ or $\Delta(\sqrt{x})$ of the function $h(x)$ depend on the measured mean value $x_{\text {mean }}$. But otherwise, it is difficult to understand that $E_{x^{2}}$ can never, at no measurement, vanish. Moreover, $E_{x^{2}}$ will always be larger than $D_{x}$; more precisely, $E_{x^{2}} \geq D_{x}$ (see Eq. (6)). This conclusion dictates a condition for the inverse function, $\sqrt{x}$, which follows from Eq. (7); namely, $E_{y} \geq \frac{1}{2} D_{y}$. All that can be well observed in Figs. 1 and 2, where the dependences of the dispersions of the functions $D_{h}=D_{x^{2}}\left(E_{x}, D_{x}\right)$ and $D_{h}=D_{\sqrt{x}}\left(E_{x}, D_{x}\right)$, respectively, on the measured "means" of their argument $E_{x}$ (the argument $D_{x}$ is assumed to be fixed) are plotted. Note that the very possibility to obtain a graphic representaion for the obtained relations allows one to discuss the character of future measurements and plan them.

Note that, in the limiting case $D_{x}=0$, we have

$$
\begin{aligned}
& E_{x^{2}}=E_{x}^{2} ; \quad D_{x^{2}}=0 ; \\
& E_{\sqrt{x}}=\sqrt{E_{x}} ; \quad D_{\sqrt{x}}=0 ;
\end{aligned}
$$

But the "usual" rules of error propagation

$E_{x^{2}}=E_{x}^{2}, \quad E_{\sqrt{x}}=\sqrt{E_{x}}$

can be applied in this case as well. In other cases, when $D_{x}$ appreciably differs from zero, expressions (6) and (7) for $E_{x^{2}}$ provide more proper values.

\section{Conclusions}

Relations (6) and (7) give a correct result for samples and can be widely used to reduce and to substantially simplify computation procedures, when carrying out 
statistical calculations for the functions $x^{2}$ and $\sqrt{x}$. In the case where the initial array of experimental data is absent, the method of error propagation may turn out a unique rather simple, but correct way to calculate $E_{h}, D_{h}$, and the variance $\sigma$ for the indicated functions. Since the dispersions $D_{h}$ and the variances $\sigma$ for both examined functions practically coincide with the corresponding real values, the exact propagation of errors is possible for a chain of functions of the type $x^{2}$ and $\sqrt{x}$ or, taking the results of work [2] into account, any other combination of the functions $x^{2}$, $\sqrt{x}, \cos x$, and $\arccos x$.

Therefore, on the basis of the obtained analytical relations, two simple universal algorithms can be constructed for the calculation of pairs of separate values: $\left(E\left(x^{2}\right), D\left(x^{2}\right)\right)$ and $(E(\sqrt{x}), D(\sqrt{x}))$. Those algorithms can be inbuilt as separate modules (subroutines) into any software codes, with the algorithms remaining transparent (easy for reading) at that. This is impossible in principle for other propagation methods, because the latter demand that the superposition of functions should be expanded in series (or differentiated) as a whole. Therefore, a separate procedure has to be constructed for each individual problem.

The error of a function can be predicted, and its dependence on the planned region of measurements of a physical quantity can be plotted.

Also interesting is a possibility to obtain an exact mean shift for $E\left(x^{2}\right)$ and $E(\sqrt{x})$. In the examples presented above, this shift did not affect the mean values and did not play any role. However, in some applications, it does exist, and its value can be used.

\section{APPENDIX}

In this Appendix, the validity of the relations obtained for two functions, $E\left(x^{2}\right)$ and $E(\sqrt{x})$, i.e. the reduction of the integral equations (4) and (5) to tabulated integrals and the reduction of the obtained relations to the convenient forms (6) and (7), is proved mathematically.

\section{Mathematical expectation}

\section{$E_{h}$ for the function $h(x)=x^{2}$}

In the case $h(x)=x^{2}$, Eq. (4), taking the Gaussian distribution (3) into account, reads

$$
\begin{aligned}
& E_{h}=E_{x^{2}}=\int_{-\infty}^{\infty} x^{2} f(x) d x=\int_{-\infty}^{\infty} x^{2} \sqrt{\frac{p}{\pi}} \exp \left[-p(x-\mu)^{2}\right]= \\
& =\sqrt{\frac{p}{\pi}} \int_{-\infty}^{\infty} x^{2} \exp \left[-p x^{2}+2 p x \mu-p \mu^{2}\right] d x=
\end{aligned}
$$

$=\sqrt{\frac{p}{\pi}} \exp \left(-p \mu^{2}\right) \int_{-\infty}^{\infty} x^{2} \exp \left[-p x^{2}+2 p x \mu\right] d x=$

$=\sqrt{\frac{p}{\pi}} \exp \left(-p \mu^{2}\right) J$,

where

$J=\int_{-\infty}^{\infty} x^{2} \exp \left[-p x^{2}+2 p \mu x\right] d x$

In essence, the integral expression $J$ is the tabulated integral $[3$, formula $(3.462 .8)]$

$T_{8}=\int_{-\infty}^{\infty} x^{2} \exp \left[-p x^{2}+2 q x\right] d x=\frac{1}{2 p} \sqrt{\frac{\pi}{p}}\left(1+2 \frac{q^{2}}{p}\right) \exp \left(\frac{q^{2}}{p},\right)$.

in which $q=p \mu$. Substituting this value into the expression for $T_{8}$, we have

$J=\frac{1}{2 p} \sqrt{\frac{\pi}{p}}\left(1+2 \frac{p^{2} \mu^{2}}{p}\right) \exp \left(\frac{p^{2} \mu^{2}}{p}\right)=$

$=\frac{1}{2 p} \sqrt{\frac{\pi}{p}}\left(1+2 p \mu^{2}\right) \exp \left(p \mu^{2}\right)$.

By including this expression into Eq. (10), we obtain

$E_{h}=E_{x^{2}}=\sqrt{\frac{p}{\pi}} \exp \left(-p \mu^{2}\right) J=$

$=\sqrt{\frac{p}{\pi}} \exp \left(-p \mu^{2}\right) \frac{1}{2 p} \sqrt{\frac{\pi}{p}}\left(1+2 p \mu^{2}\right) \exp \left(p \mu^{2}\right)=$

$=\frac{1}{2 p}\left(1+2 p \mu^{2}\right)=\frac{1}{2 p}+\mu^{2}$.

With regard for the relation $p=\frac{1}{2 D(x)}=\frac{1}{2 D_{x}}$ and Eq. (4), we ultimately obtain

$E_{h}=E_{x^{2}}=\mu^{2}+D_{x}=E_{x}^{2}+D_{x}$.

This is an almost expected result. The "shift" $D_{x}$ can be ignored if it is small $\left(D_{x} \approx 0\right)$, but expression $(15)$ is an exact working formula in the case $h(x)=x^{2}$.

Dispersion $D_{h}$ for the function $h(x)=x^{2}$

From Eq. (5), the "propagation error" equals

$D\left(x^{2}\right)=\int_{-\infty}^{\infty} x^{4} f(x) d x-E_{h}^{2}=J_{0}-E_{h}^{2}$.

Let us consider the integral $J_{0}$. It can be reduced to the corresponding tabulated integral, by using transformations similar to Eq. (10):

$J_{0}=\int_{-\infty}^{\infty} x^{4} f(x) d x=\int_{-\infty}^{\infty} x^{4} \sqrt{\frac{p}{\pi}} \exp \left[-p(x-\mu)^{2}\right]=$

$=\sqrt{\frac{p}{\pi}} \int_{-\infty}^{\infty} x^{4} \exp \left[-p x^{2}+2 p x \mu-p \mu^{2}\right] d x=$

ISSN 2071-0194. Ukr. J. Phys. 2017. Vol. 62, No. 2 
$=\sqrt{\frac{p}{\pi}} \exp \left(-p \mu^{2}\right) \int_{-\infty}^{\infty} x^{4} \exp \left[-p x^{2}+2 p x \mu\right] d x=$

$=\sqrt{\frac{p}{\pi}} \exp \left(-p \mu^{2}\right) J_{01}$

where

$J_{01}=\int_{-\infty}^{\infty} x^{4} \exp \left[-p x^{2}+2 p \mu x\right] d x$.

The integral expression $J_{01}$ looks like the tabulated integral $T_{2}$ [3, formula (3.462.2)],

$T_{2}=\int_{-\infty}^{\infty} x^{n} \exp \left[-p x^{2}+2 q x\right] d x=\frac{1}{2^{n-1} p} \sqrt{\frac{\pi}{p}} \frac{d^{n-1}}{d q^{n-1}}\left[q \exp \left(\frac{q^{2}}{p}\right)\right]$,

$T_{2}^{\prime}=\int_{-\infty}^{\infty} x^{n} \exp \left[-p x^{2}+2 q x\right] d x=$

$=n ! \sqrt{\frac{\pi}{p}} \exp \left(\frac{q^{2}}{p}\right)\left(\frac{q}{p}\right)^{n} \sum_{k=0}^{E\left(\frac{n}{2}\right)} \frac{1}{(n-2 k) ! k !}\left(\frac{p}{4 q^{2}}\right)^{k}$.

At $n=4$, the both integrals are identical, i.e. $T_{2}^{\prime}=T_{2}$. Hence, at $n=4$, we obtain

$J_{01}=T_{2}=\int_{-\infty}^{\infty} x^{4} \exp \left[-p x^{2}+2 q x\right] d x=$

$=\frac{1}{2^{3} p} \sqrt{\frac{\pi}{p}} \frac{d^{3}}{d q^{3}}\left[q \exp \left(\frac{q^{2}}{p}\right)\right]=\frac{1}{8 p} \sqrt{\frac{\pi}{p}} J_{02}$,

where

$J_{02}=\frac{d^{3}}{d q^{3}}\left[q \exp \left(\frac{q^{2}}{p}\right)\right]=\frac{d^{2}}{d q^{2}}\left[\exp \left(\frac{q^{2}}{p}\right)+\frac{2 q^{2}}{p} \exp \left(\frac{q^{2}}{p}\right)\right]=$

$=\frac{d}{d q}\left[\frac{2 q}{p} \exp \left(\frac{q^{2}}{p}\right)+\frac{4 q}{p} \exp \left(\frac{q^{2}}{p}\right)+\frac{4 q^{3}}{p^{2}} \exp \left(\frac{q^{2}}{p}\right)\right]=$

$=\frac{d}{d q}\left[\frac{6 q}{p} \exp \left(\frac{q^{2}}{p}\right)+\frac{4 q^{3}}{p^{2}} \exp \left(\frac{q^{2}}{p}\right)\right]=$

$=\left[\frac{6}{p} \exp \left(\frac{q^{2}}{p}\right)+\frac{12 q^{2}}{p^{2}} \exp \left(\frac{q^{2}}{p}\right)+\frac{12 q^{2}}{p^{2}} \exp \left(\frac{q^{2}}{p}\right)+\right.$

$\left.+\frac{8 q^{4}}{p^{3}} \exp \left(\frac{q^{2}}{p}\right)\right]=\left[\frac{6}{p}+\frac{24 q^{2}}{p^{2}}+\frac{8 q^{4}}{p^{3}}\right] \exp \left(\frac{q^{2}}{p}\right)$.

Substituting $q=p \mu$, we obtain

$J_{02}=\left[\frac{6}{p}+\frac{24 p^{2} \mu^{2}}{p^{2}}+\frac{8 p^{4} \mu^{4}}{p^{3}}\right] \exp \left(\frac{p^{2} \mu^{2}}{p}\right)=$

$=\left[\frac{6}{p}+24 \mu^{2}+8 p \mu^{4}\right] \exp \left(p \mu^{2}\right)$.

Accordingly,

$J_{01}=\frac{1}{8 p} \sqrt{\frac{\pi}{p}} J_{02}=\frac{1}{8 p} \sqrt{\frac{\pi}{p}}\left[\frac{6}{p}+24 \mu^{2}+8 p \mu^{4}\right] \exp \left(p \mu^{2}\right)=$

$=\sqrt{\frac{\pi}{p}} \exp \left(p \mu^{2}\right)\left[\frac{3}{4 p^{2}}+3 \frac{\mu^{2}}{p}+\mu^{4}\right]$.

ISSN 2071-0194. Ukr. J. Phys. 2017. Vol. 62, No. 2
Substituting this value into Eq. (13) and taking into account that $p=\frac{1}{2 D_{x}}$, we have

$J_{0}=\sqrt{\frac{p}{\pi}} \exp \left(-p \mu^{2}\right) J_{01}=\sqrt{\frac{p}{\pi}} \exp \left(-p \mu^{2}\right) \sqrt{\frac{\pi}{p}} \exp \left(p \mu^{2}\right) \times$

$\times\left[\frac{3}{4 p^{2}}+3 \frac{\mu^{2}}{p}+\mu^{4}\right]=\frac{3}{4 p^{2}}+3 \frac{\mu^{2}}{p}+\mu^{4}=$

$=\frac{3}{4} 4 D_{x}^{2}+3 \mu^{2} 2 D_{x}+\mu^{4}=3 D_{x}^{2}+6 \mu^{2} D_{x}+\mu^{4}$.

Making allowance for $J_{0}$ and Eq. (12), we obtain for the dispersion $D\left(x^{2}\right)$ of the function $h(x)=x^{2}$ that

$D\left(x^{2}\right)=J_{0}-\chi^{2}=3 D_{x}^{2}+6 \mu^{2} D_{x}+\mu^{4}-\left(\mu^{2}+D_{x}\right)^{2}=$

$=3 D_{x}^{2}+6 \mu^{2} D_{x}+\mu^{4}-\mu^{4}-2 \mu^{2} D_{x}-D_{x}^{2}=$

$=2 D_{x}^{2}+4 \mu^{2} D_{x}=2 D_{x}^{2}+4 E_{x}^{2} D_{x}$.

The final result looks like

$D\left(x^{2}\right)=2 D_{x}^{2}+4 \mu^{2} D_{x}=2 D_{x}^{2}+4 E_{x}^{2} D_{x}$.

This is the "error propagation" rule for the function $h(x)=x^{2}$.

It should be noted that all the used mathematical operations (the calculation of an integral of a sum as a sum of integrals, factorization, and so on) are proper from the viewpoint of statistical rules [1].

\section{Mathematical expectation $\boldsymbol{E}_{h}$} and dispersion $D_{h}$ for the function $h(x)=\sqrt{x}$

A direct calculation of $E_{\sqrt{x}}$ and $D_{\sqrt{x}}$ with the help of tabulated integrals is rather a problematic task. The required relations can be obtained by considering the function $\sqrt{x}$ as an inverse one to $x^{2}$ and by applying formulas (6). Really, the latter give us the explicit relations between four integrals $E_{x}, D_{x}, E_{x^{2}}$, and $D_{x^{2}}$; or, roughly speaking, between four numbers:

$E_{x^{2}}=E_{x^{2}}\left(E_{x}, D_{x}\right), \quad D_{x^{2}}=D_{x^{2}}\left(E_{x}, D_{x}\right)$.

The inverse functions

$E_{x}=E_{x}\left(E_{x^{2}}, D_{x^{2}}\right), \quad D_{x}=D_{x}\left(E_{x^{2}}, D_{x^{2}}\right)$

determined from Eqs. (6) and (15) must also correctly describe mathematical relations between those four integrals. If $E_{x^{2}}$ and $D_{x^{2}}$ are obtained in a different way (e.g., if they are measured) and have the same numerical values as those calculated by Eq. (15), they will satisfy the coupling equations for four integrals (6) and (15), if and only if $E_{x}$ and $D_{x}$ have the same values given in Eq. (6).

In other words, if $y=x^{2}$ and, accordingly, $x=\sqrt{y}$, the relations $E_{x}=E_{x}\left(E_{y}, D_{y}\right)$ and $D_{x}=D_{x}\left(E_{y}, D_{y}\right)$, which are inverse to Eqs. (6) and (15), give us the correct values of integral expressions for the mathematical expectation $E_{x}$ and dispersion $D_{x}$ determined by formulas (8) and (9) for a function of the random variable $y$, which is coupled with the variable $x$ by the law $y=x^{2}$ or $x=\sqrt{y}$. This means that, by solving Eqs. (6) and (15) for $x$, we can use simple calculations to obtain $E_{x}$ and $D_{x}$ on the basis of $E_{y}$ - and $D_{y}$-values, which are means for the measurements of the random variable $y$, which is coupled with $x$ by the relation $x=\sqrt{y}$. 
Let us solve Eqs. (6). For this purpose, let us rewrite them in the form

$E_{y}=E_{x}^{2}+D_{x}$,

$D_{y}=2 D_{x}^{2}+4 E_{x}^{2} D_{x}$.

Bearing in mind that the integrals $E_{y}$ and $D_{y}$ are coupled by the function $y=x^{2}$, and the integrals $E_{x}$ and $D_{x}$ by the function $x=\sqrt{y}$, let us solve those equations with respect to the integrals $E_{x}$ and $D_{x}$. Squaring the both sides of Eq. (16) and multiplying them by 2 , we obtain

$2 E_{y}^{2}=2\left(E_{x}^{2}+D_{x}\right)^{2}$.

Subtracting this equation from Eq. (17), we have

$D_{y}-2 E_{y}^{2}=2 D_{x}^{2}+4 E_{x}^{2} D_{x}-\left[2\left(E_{x}^{2}+D_{x}\right)^{2}\right]=$

$=2 D_{x}^{2}+4 E_{x}^{2} D_{x}-2 E_{x}^{4}-4 E_{x}^{2} D_{x}-2 D_{x}^{2}=-2 E_{x}^{4}$.

From whence, we obtain the explicit expression for the function $E_{x}\left(E_{y}, D_{y}\right)$ :

$E_{x}^{4}=E_{y}^{2}-\frac{1}{2} D_{y}$.

Writing down Eq. (16) in the form $D_{x}=E_{y}-E_{x}^{2}$ and substituting the value $E_{x}^{2}=\sqrt{E_{y}^{2}-\frac{1}{2} D_{y}}$ obtained from Eq. (6) (here, the sign "+" before the root was selected taking into account that $E_{x}^{2} \geq 0$ ), we ultimately obtain the explicit form for the function $D_{x}\left(E_{y}, D_{y}\right)$ :

$D_{x}=E_{y}-\sqrt{E_{y}^{2}-\frac{1}{2} D_{y}}$.

It should be noted that the executed transformations do not contradict statistical rules [1], i.e. they are correct.

Finally, let us rewrite the obtained relations (16)-(19) in a more clear symbolic form,

$$
\begin{aligned}
& E_{h}=E_{x^{2}}=E_{x}^{2}+D_{x} ; \\
& D_{h}=D_{x^{2}}=2 D_{x}^{2}+4 E_{x}^{2} D_{x} ; \\
& E_{h}^{4}=E_{\sqrt{x}}^{4}=E_{x}^{2}-\frac{1}{2} D_{x} ; \\
& D_{h}=D_{\sqrt{x}}=E_{x}-\sqrt{E_{x}^{2}-\frac{1}{2} D_{x}},
\end{aligned}
$$

where $x$ stands for the measured physical quantity (argument), and $h$ for the corresponding function $\left(x^{2}\right.$ or $\left.\sqrt{x}\right)$.

With regard for the results of work [2], it is useful to present the "analytical" propagation rules for two more functions: $\cos x$ and $\arccos x$. They look like

$$
\begin{aligned}
& E_{h}=E_{\cos }=\exp \left(-\frac{D_{x}}{2}\right) \cos E_{x} \\
& D_{h}=D_{\cos }=\frac{1}{2}\left[1-\exp \left(-D_{x}\right)\right]\left[1-\exp \left(-D_{x}\right) \cos 2 E_{x}\right] \\
& E_{h}=E_{\arccos }=\arccos \frac{E_{x}}{ \pm \sqrt{E_{x}^{2}+\sqrt{\left(1-E_{x}^{2}\right)^{2}-2 D_{x}}}} \\
& D_{h}=D_{\arccos }=\ln \left(\frac{1}{E_{x}^{2}+\sqrt{\left(1-E_{x}^{2}\right)^{2}-2 D_{x}}}\right)
\end{aligned}
$$

respectively. Considering the formalization $x \approx E_{x}, \quad k(\Delta x)^{2} \approx D_{x}$,

Eqs. (20) and (21) give us the required "propagation rules" for the means and errors in the case of the functions $x^{2}, \sqrt{x}, \cos x$, and $\arccos x$ :

$X \rightarrow H, \quad|\Delta X| \rightarrow|\Delta H|$.

The presented material was reported at the seminars of eight departments at the Institute of Physics of the NASU. The author is sincerely grateful to the teams and the heads of those departments (I.V.Blonskyi, M.S. Brodyn, A.G. Naumovets, A.M.Negryiko, Yu.O.Reznikov, S.M.Ryabchenko, P.M. Tomchuk, and L.P. Yatsenko) for their attention. There is no doubt that, without critical remarks made during the discussions at the seminars, the work would have been considerably worse or would have not been carried out at all. My special thanks are due to S.M. Ryabchenko for his extremely critical discussion of the results obtained, to G.V.Klimusheva for her reading of the manuscript and critical remarks, and to O.I. Voitenko for his advice. I am also grateful to everybody who was not indifferent to this work and supported it.

1. D.J. Hudson, Statistics. Lectures on Elementary Statistics and Probability (CERN, 1964).

2. G.G. Rode. The propagation of measurement errors and measured means of a physical quantity for the elementary functions $\cos x$ and $\arccos x$. Ukr. J. Phys. 61, 345 (2016) [DOI: 10.15407/ujpe61.04.0345].

3. I.S. Gradshtein, I. M. Ryzhik. Table of Integrals, Series, and Products (Academic Press, 1980) [ISBN: 0122947606].

4. Propagation of uncertainty (Wikipedia) [https:// en.wikipedia.org/wiki/Propagation of uncertainty].

5. H.H. Ku. Notes on the use of propagation of error formulas. J. Res. Nat. Bur. Stand. 70C, 263 (1966) [DOI: 10.6028/ jres.070C.025].

6. Ph.R. Bevington, D.K. Robinson. Data Reduction and Error Analysis for the Physical Sciences (McGraw-Hill, 2002) [ISBN: 0-07-247227-8].

7. J.R. Taylor. An Introduction to Error Analysis: The Study of Uncertainties in Physical Measurements (University Science Books, 1997)[ISBN: 0-935702-75X].

8. B.N. Taylor, C.E. Kuyatt. Guidelines for Evaluating and Expressing the Uncertainty of NIST Measurement Results (NIST Technical Note 1297) (National Institute of Standards and Technology, 1994).

9. P.K. Sinervo. Definition and treatment of systematic uncertainties in high energy physics and astrophysics. In Proceedings of the PHYSTAT2003 Conference, SLAC, Stanford, CA, September 8-11 (2003), p. 122.

10. J. Denker. Nonlinear least squares [http://www.av8n.com/ physics/nonlinear-least-squares.htm].

ISSN 2071-0194. Ukr. J. Phys. 2017. Vol. 62, No. 2 
11. E.W. Weisstein. Standard deviation entry at mathworld. [http://mathworld.wolfram.com/StandardDeviation.html].

12. Evaluation of measurement data - An introduction to the "Guide to the expression of uncertainty in measurement" and related documents. http://www.bipm.org/utils/ common/documents/jcgm/JCGM 1042009 E.pdf.

13. D.M. Kheiker, E.L. Lube, A. $\bar{V}$. Mirenskii, N.I. Komyak, O.V. Maklakov, L.Z. Tatkin, E.N. Gurevich, V.S. Rogachev. A design of automatic diffractometer for studying single crystals. In Equipment and Methods of X-ray Analysis (Mashinostroenie, 1968), Vol. 3, p. 130 (in Russian).

14. E.L. Lube, D.M. Kheiker. A control program for the automatic diffractometer DAR-1. In Equipment and Methods of X-ray Analysis (Mashinostroenie, 1968), Vol. 3, p. 145 (in Russian).

Received 23.03.16.

Translated from Ukrainian by O.I. Voitenko
Г.Г. Роде

\section{ПЕРЕНОС ПОХИБОК}

ТА СЕРЕДНІХ ВИМІРІВ ФІЗИЧНОЇ ВЕЛИЧИНИ ДЛЯ ЕЛЕМЕНТАРНИХ ФУНКЦІЙ $x^{2}$ ТА $\sqrt{x}$

$\mathrm{P}$ е $з$ ю м е

Отримані "правила переносу похибки та середнього" однієї вимірюваної фізичної величини на іншу, пов'язану з нею функційним зв'язком $x^{2}$ або $\sqrt{x}$. В ці правила по природі закладена вагова схема Гауса. Тому вони мають добре працювати в рамках реальної вагової схеми Гауса 3 дискретними даними реального фізичного дослідження (з "вибірками"). Аналітична форма, в якій представлені згадані правила ("аналітичні правила переносу"), а також їх точний характер дозволяє спростити і прискорити процедуру обробки й аналізу експериментальних даних. 